\title{
Solvent Polarity Effect on Nonradiative Decay Rate of Thioflavin T
}

\author{
Vitali I. Stsiapura, ${ }^{1}$ Sergey A. Kurhuzenkau, ${ }^{1}$ Valery A. Kuzmitsky, ${ }^{2}$ Oleg V. Bouganov, ${ }^{3}$ \\ Sergey A. Tikhomirov ${ }^{3}$
}

${ }^{1}$ Yanka Kupala State University, Grodno, Belarus; ${ }^{2}$ Institute for Command Engineers of the Ministry for Emergencies of the Republic of Belarus, Minsk, Belarus; ${ }^{3}$ Institute of Physics, National Academy of Sciences of Belarus, Minsk, Belarus

\section{Contents}

Description of fluorescence quantum yield measurements.

Figure SI1. Absorption, fluorescence spectra of ThT in 1-butanol at $298 \mathrm{~K}$.

Figure SI2. Absorption, fluorescence spectra of ThT in DMF at $298 \mathrm{~K}$.

Figure SI3. Absorption, fluorescence spectra of ThT in pyridine at $298 \mathrm{~K}$. S3

Figure SI4. Absorption, fluorescence spectra of ThT in acetonitrile at $298 \mathrm{~K}$. S4

Figure SI5. Absorption, fluorescence spectra of ThT in acetone at $298 \mathrm{~K}$. S4

Figure SI6. Transient absorption spectra of ThT in acetonitrile S5

Figure SI7. Transient absorption spectra of ThT in methanol S5

Figure SI8. Exponential-associated spectra of transient absorption for ThT in acetone S6

Figure SI9. Exponential-associated spectra of transient absorption for ThT in methanol S6

Figure SI10. Exponential-associated spectra of transient absorption for ThT in acetonitrile

Figure SI11. Exponential-associated spectra of transient absorption for ThT in

N,N-dimethylformamide

Figure SI12. Exponential-associated spectra of transient absorption for ThT in pyridine

Figure SI13. Rate constants ratio $k_{\mathrm{nr}} / k_{\mathrm{r}}$ dependence on $(\varepsilon-1) /(2 \varepsilon+1)$ at $\eta / \mathrm{T}=2$ and $3 \mu \mathrm{Pa} \cdot \mathrm{s} / \mathrm{K}$ for ThT in different solvents

Figure SI14. Absorbance spectrum of ThT in aqueous solution.

Invariance of electron transition properties for charged molecule relative to a coordinate origin displacement 


\section{Description of fluorescence quantum yield measurements}

We followed Williams' technique[1] while measuring fluorescence quantum yields of ThT at 298K in polar solvents. At least 5 samples with different concentrations of the dye in a particular solvent were used to measure absorbance and fluorescence spectra. We determined slope coefficients $\left(\operatorname{grad}_{\mathbf{x}}\right)$ from linear dependency of the integrated emission intensity vs absorbance, and quantum yields $\Phi$ were calculated using

$$
\Phi_{x}=\Phi_{s t} \frac{\operatorname{grad}_{x}}{\operatorname{grad}_{s t}}\left(\frac{n_{x}}{n_{s t}}\right)^{2},
$$

where ST and X denote standard and test respectively. grad - the gradient (slope coefficient $b$ in Figs. SI1-SI5) from the plot of integrated fluorescence intensity vs absorbance, and $\mathbf{n}$ - the refractive index of the solvent.

A

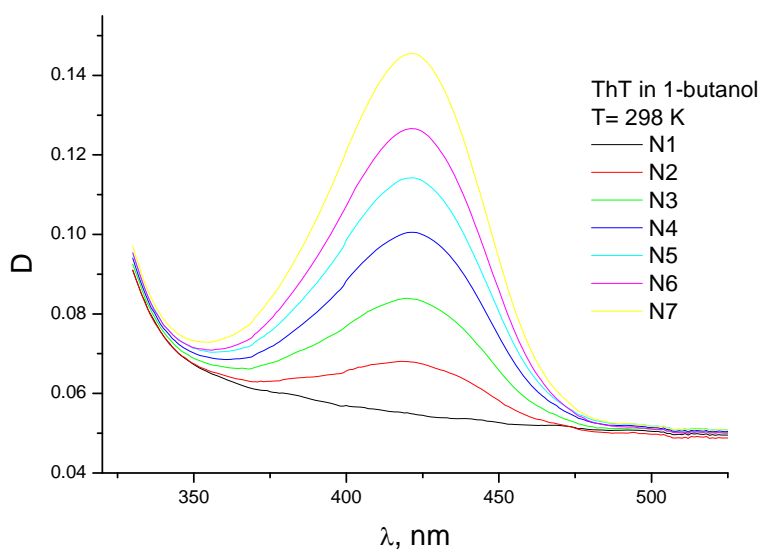

B

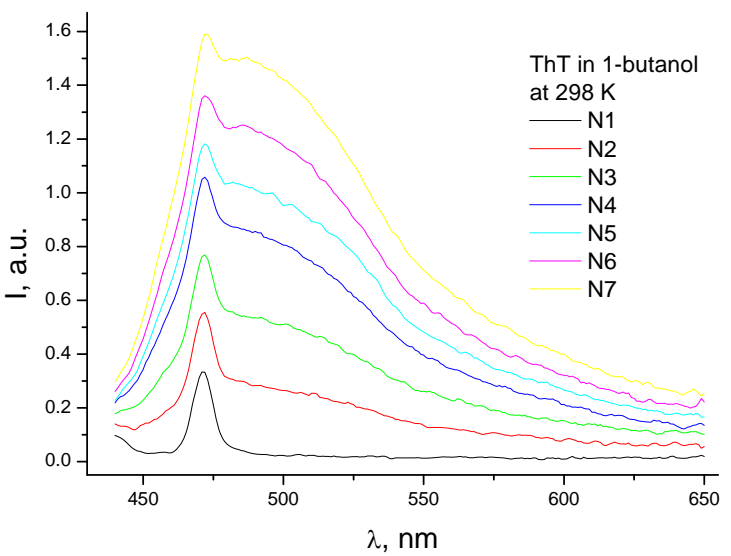

C

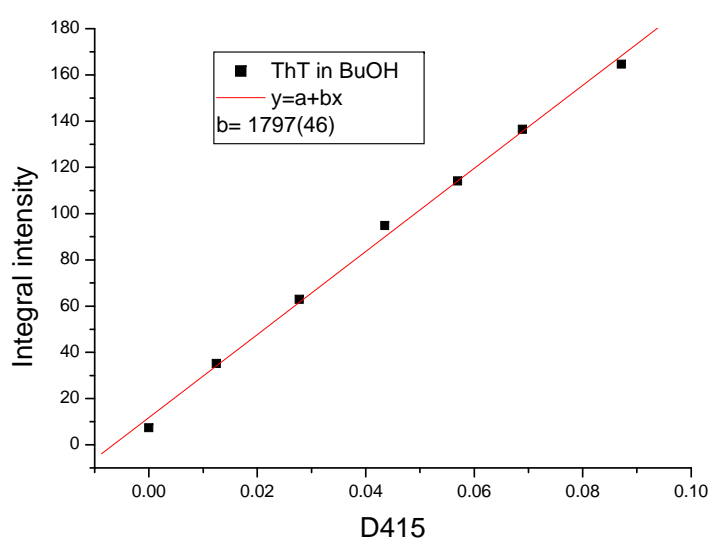

Fig.SI1. Absorption (A), fluorescence (B) spectra of ThT in 1-butanol at $298 \mathrm{~K}$. Plot of integral emission dependence (C) on absorbance at $415 \mathrm{~nm}$. Sample N1 corresponds to solvent without ThT adding 
A



B

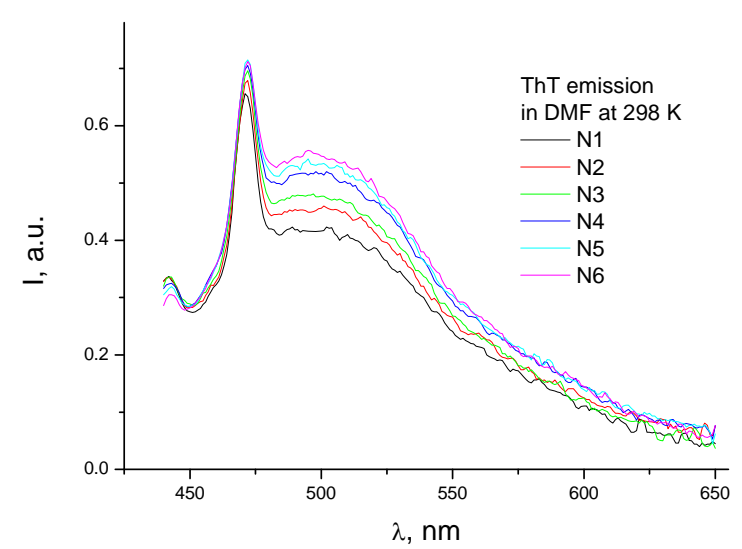

C

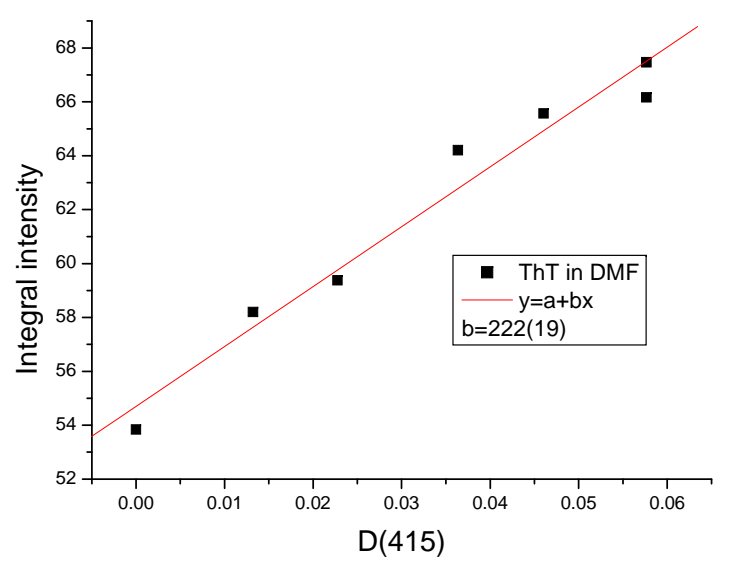

Fig.SI2. Absorption (A), fluorescence (B) spectra of ThT in N,N-dimethylformamide at $298 \mathrm{~K}$. Plot of integral emission dependence (C) on absorbance at $415 \mathrm{~nm}$. Sample N1 corresponds to solvent without ThT adding

A

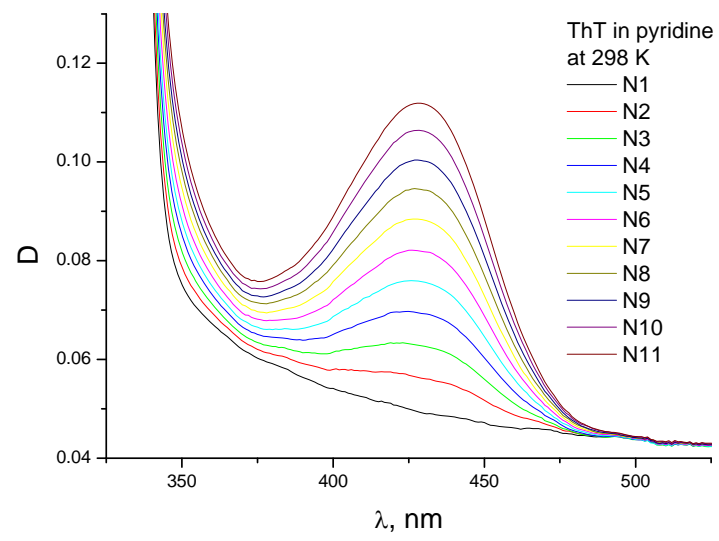

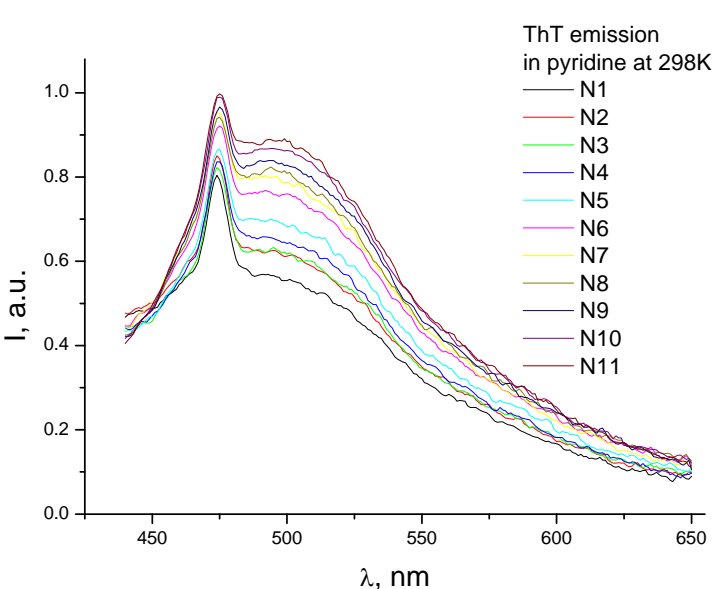

C

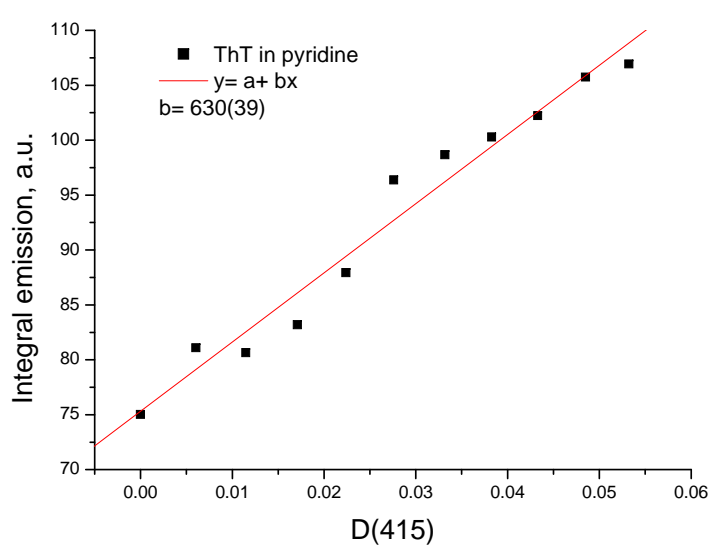

Fig.SI3. Absorption (A), fluorescence (B) spectra of ThT in pyridine at $298 \mathrm{~K}$. Plot of integral emission dependence (C) on absorbance at $415 \mathrm{~nm}$. Sample N1 corresponds to solvent without ThT adding 
A



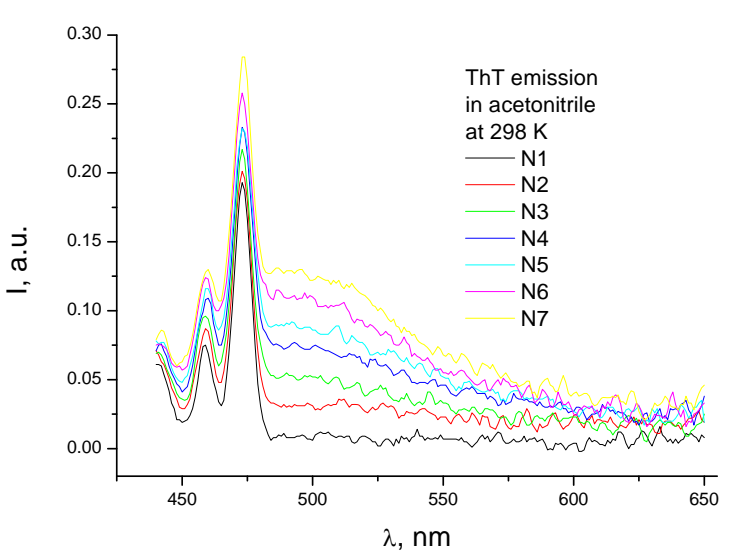

C

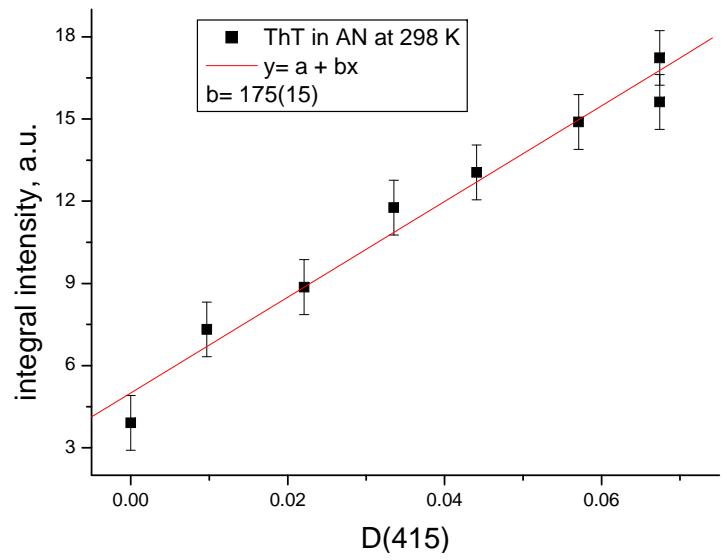

Fig.SI4. Absorption (A), fluorescence (B) spectra of ThT in acetonitrile at $298 \mathrm{~K}$. Plot of integral emission dependence (C) on absorbance at $415 \mathrm{~nm}$. Sample N1 corresponds to solvent without ThT adding

A

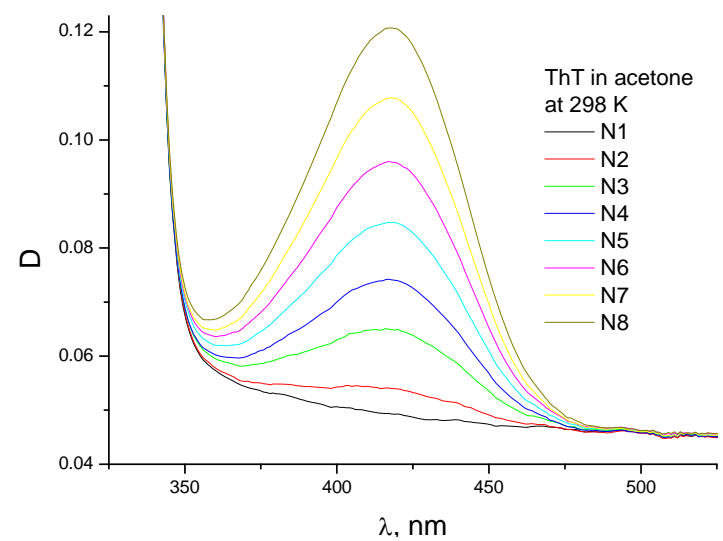

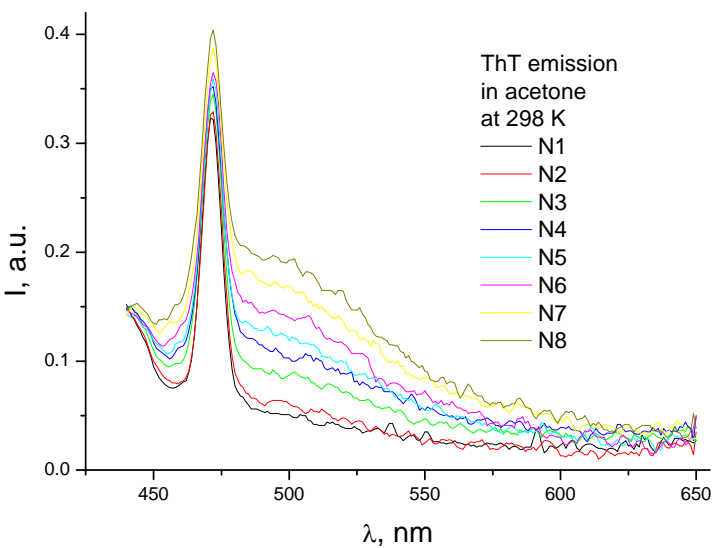

C

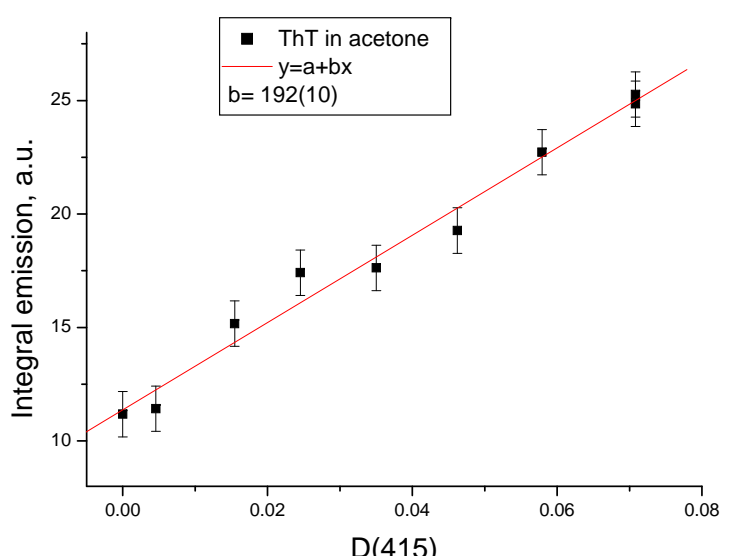

Fig.SI5. Absorption (A), fluorescence (B) spectra of ThT in acetone at $298 \mathrm{~K}$. Plot of integral emission dependence (C) on absorbance at $415 \mathrm{~nm}$. Sample N1 corresponds to solvent without ThT adding 


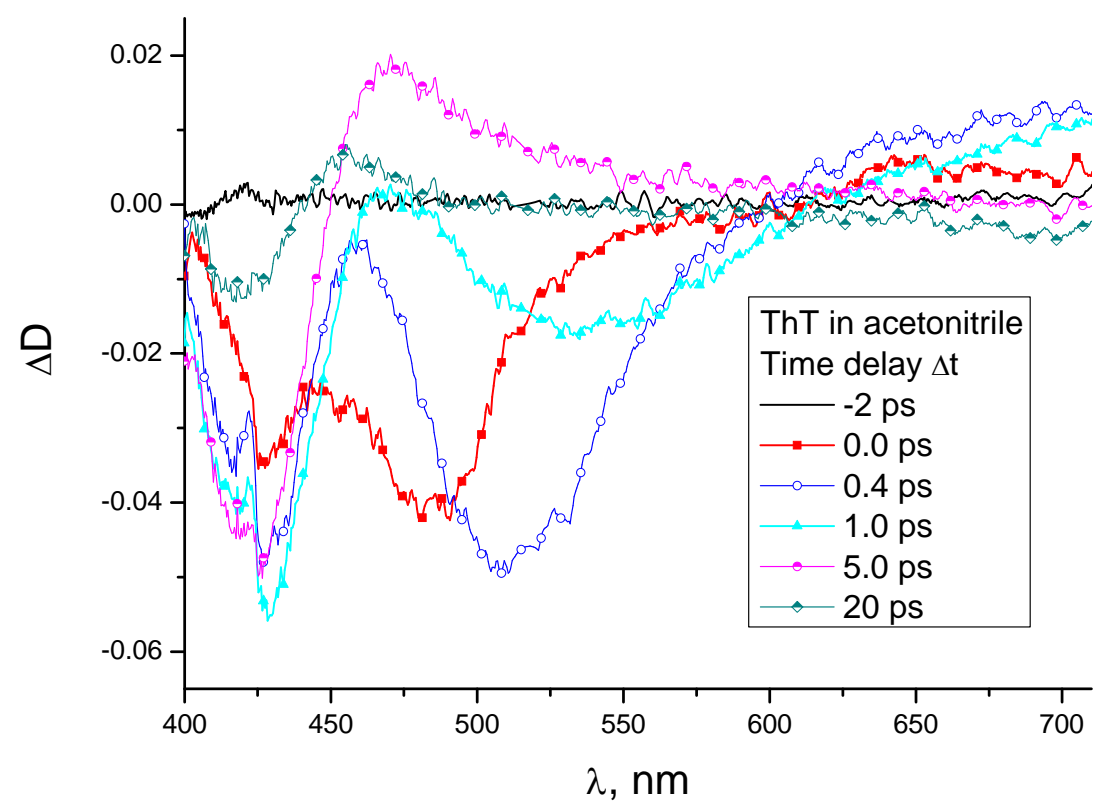

Figure SI6. Transient absorption spectra of ThT in acetonitrile at different time delays after excitation. $\lambda_{\mathrm{ex}}=400 \mathrm{~nm}, \mathrm{~T}=298 \mathrm{~K}$

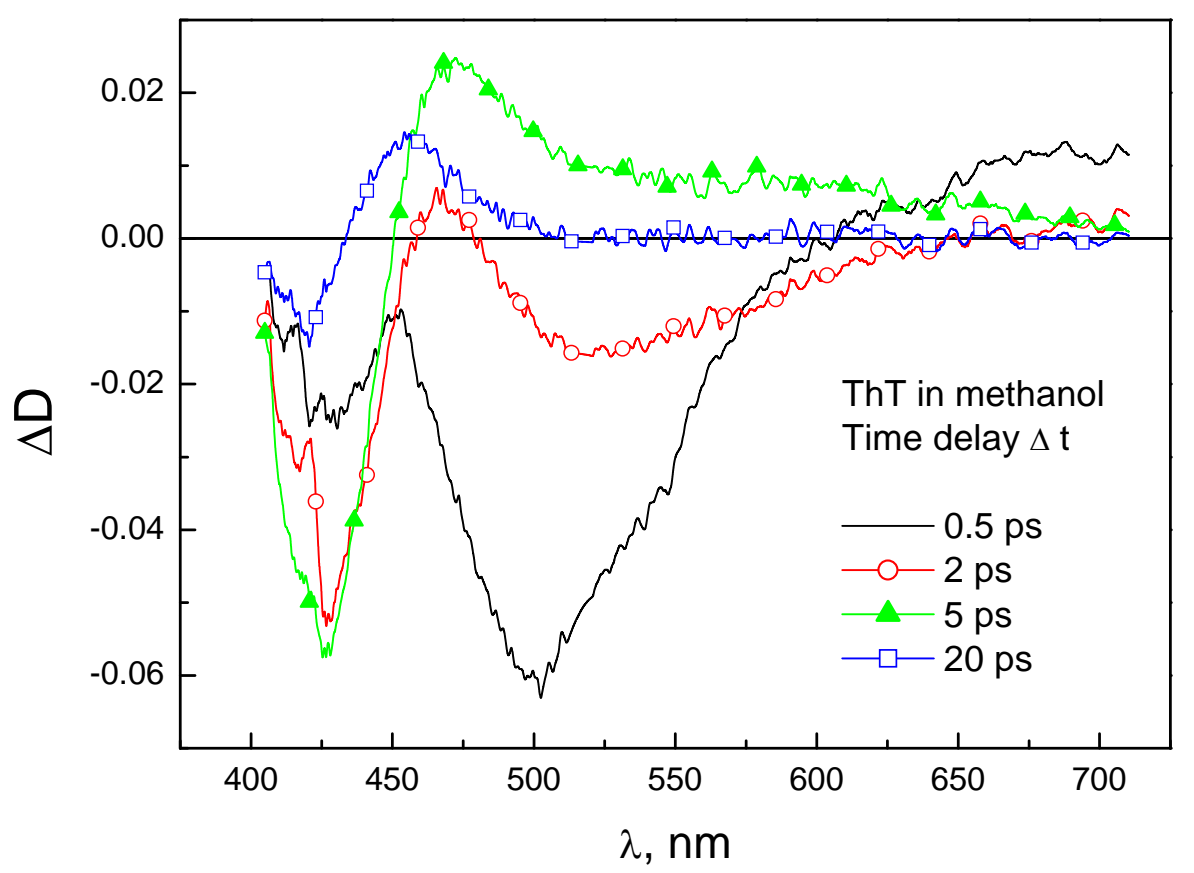

Figure SI7. Transient absorption spectra of ThT in methanol at different time delays after excitation. $\lambda_{\mathrm{ex}}=390 \mathrm{~nm}, \mathrm{~T}=298 \mathrm{~K}$ 


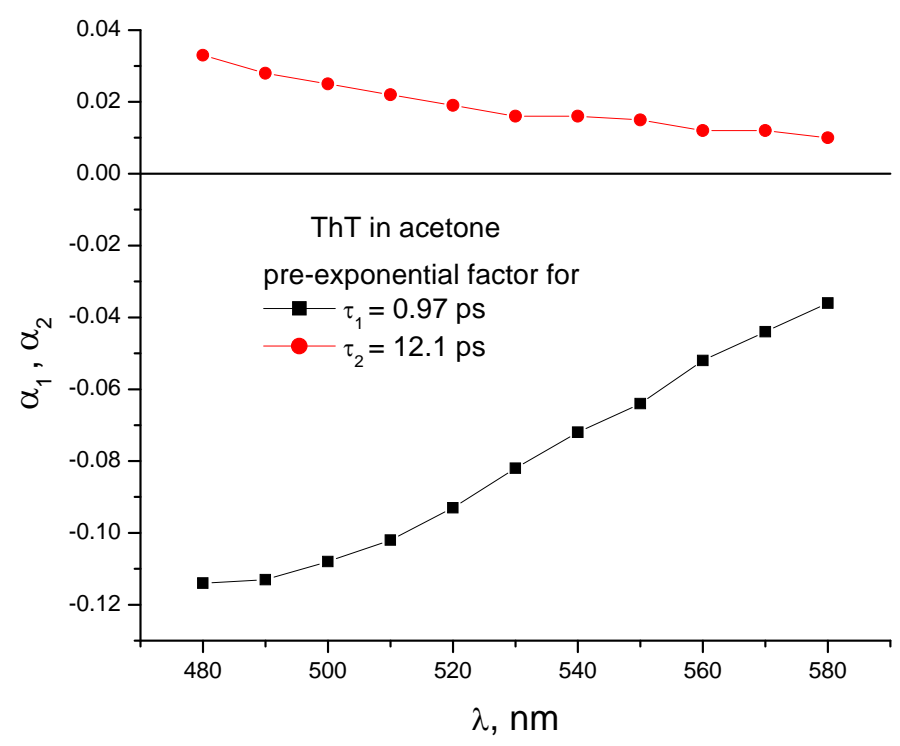

Figure SI8. Exponential-associated spectra of transient absorption for ThT in acetone. Parameters were obtained using global analysis of data with shared lifetimes. $\lambda_{\mathrm{ex}}=400 \mathrm{~nm}$, $\mathrm{T}=298 \mathrm{~K}$



Figure SI9. Exponential-associated spectra of transient absorption for ThT in methanol. Parameters were obtained using global analysis of data with shared lifetimes. $\lambda_{\text {ex }}=390 \mathrm{~nm}$, $\mathrm{T}=298 \mathrm{~K}$ 


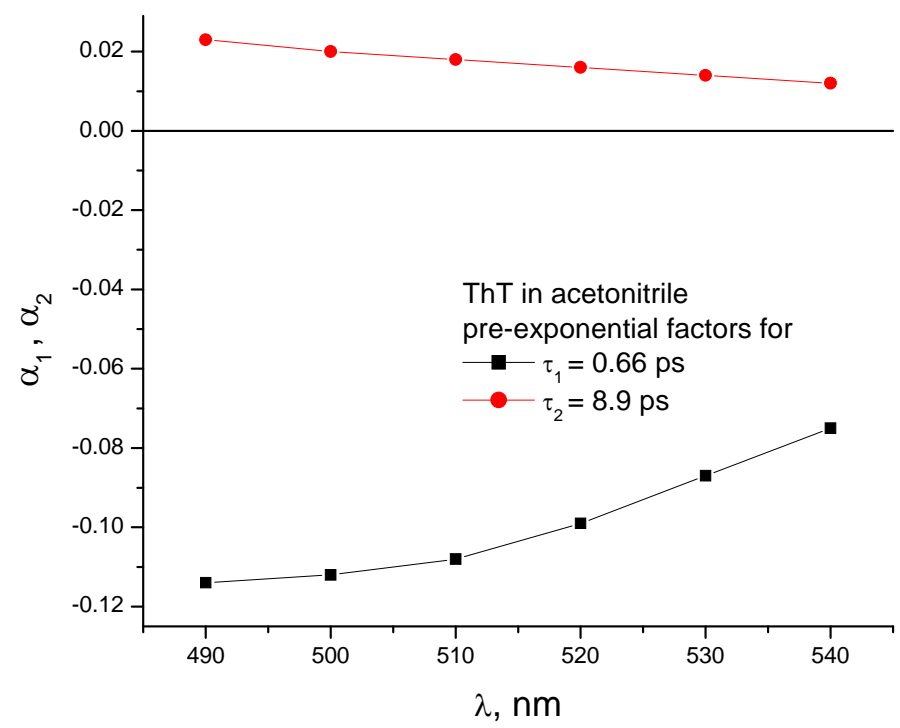

Figure SI10. Exponential-associated spectra of transient absorption for ThT in acetonitrile. Parameters were obtained using global analysis of data with shared lifetimes. $\lambda_{\mathrm{ex}}=400 \mathrm{~nm}$, $\mathrm{T}=298 \mathrm{~K}$

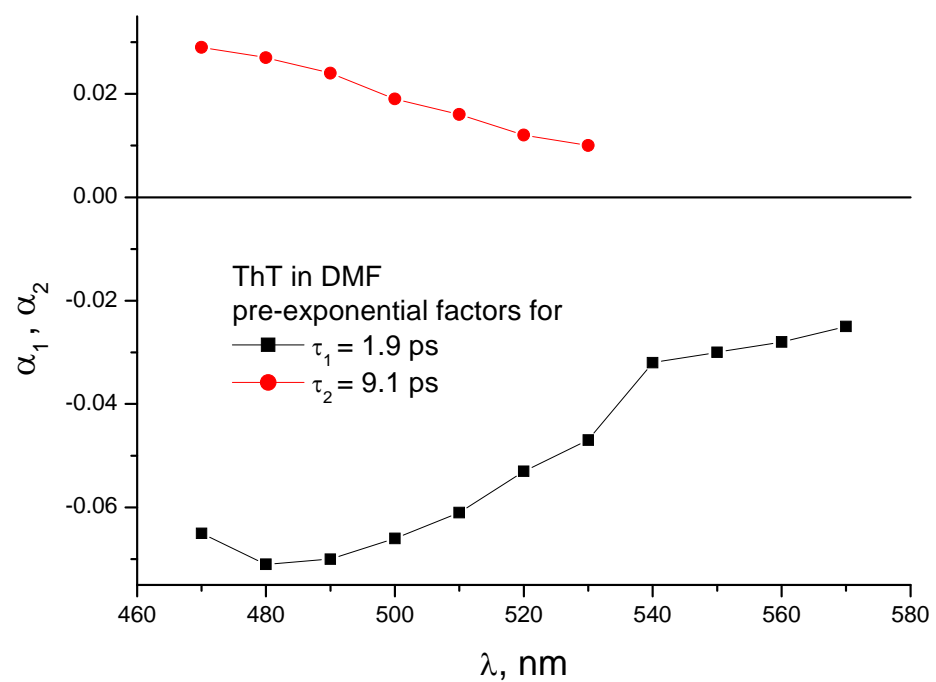

Figure SI11. Exponential-associated spectra of transient absorption for ThT in N,Ndimethylformamide. Parameters were obtained using global analysis of data with shared lifetimes. $\lambda_{\mathrm{ex}}=400 \mathrm{~nm}, \mathrm{~T}=298 \mathrm{~K}$ 


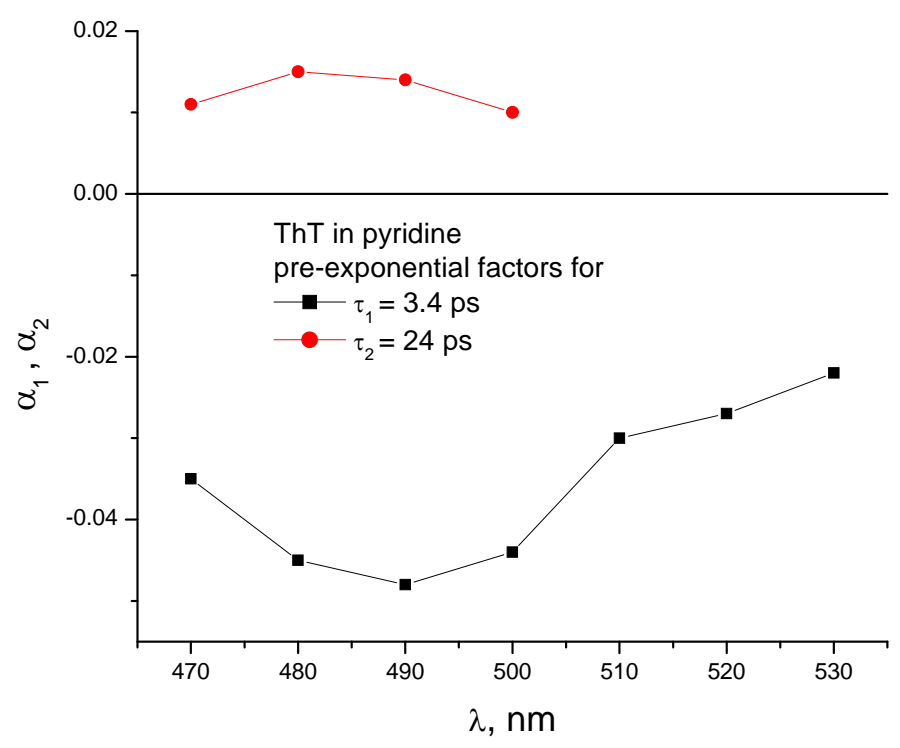

Figure SI12. Exponential-associated spectra of transient absorption for ThT in pyridine. Parameters were obtained using global analysis of data with shared lifetimes. $\lambda_{\text {ex }}=400 \mathrm{~nm}$, $\mathrm{T}=298 \mathrm{~K}$

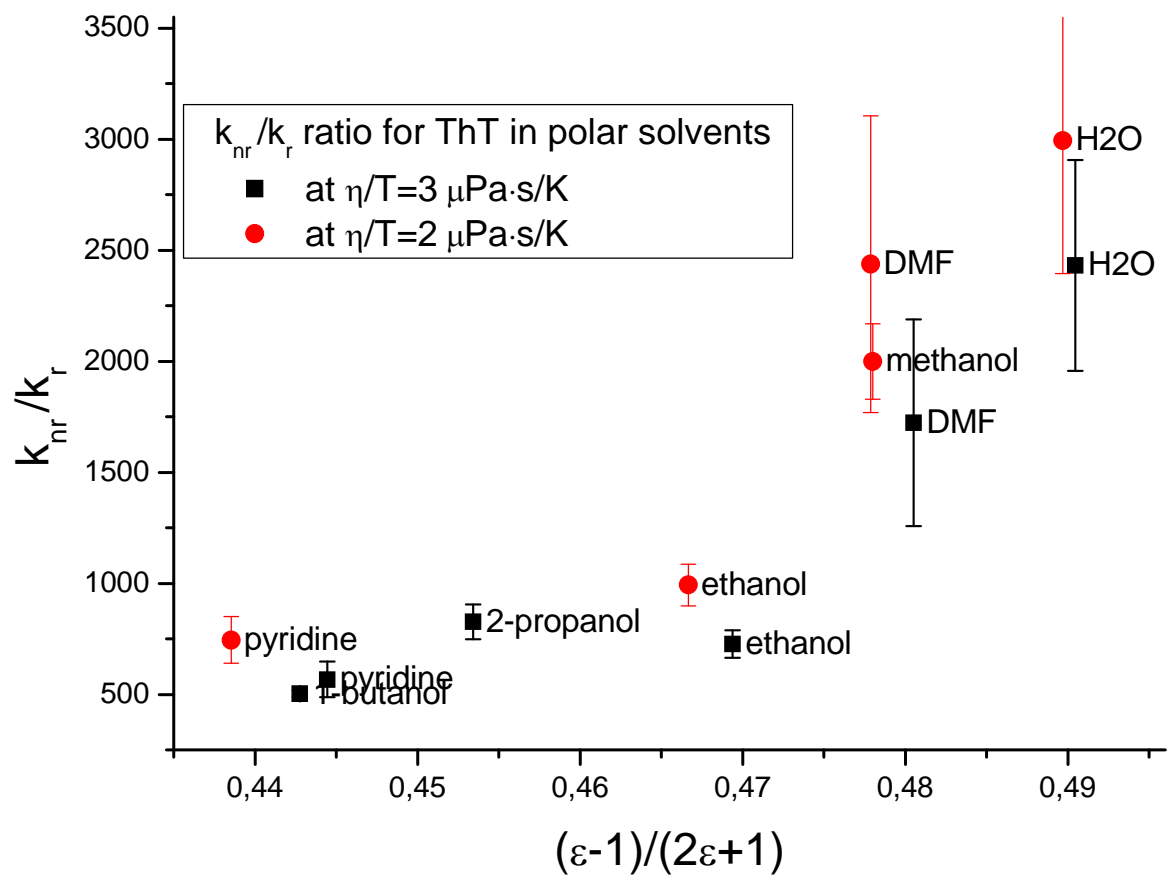

Figure SI13. Rate constants ratio $k_{\mathrm{nn}} / k_{\mathrm{r}}$ dependence on $(\varepsilon-1) /(2 \varepsilon+1)$ at $\eta / \mathrm{T}=2$ and $3 \mu \mathrm{Pa} \cdot \mathrm{s} / \mathrm{K}$ for ThT in different solvents. 


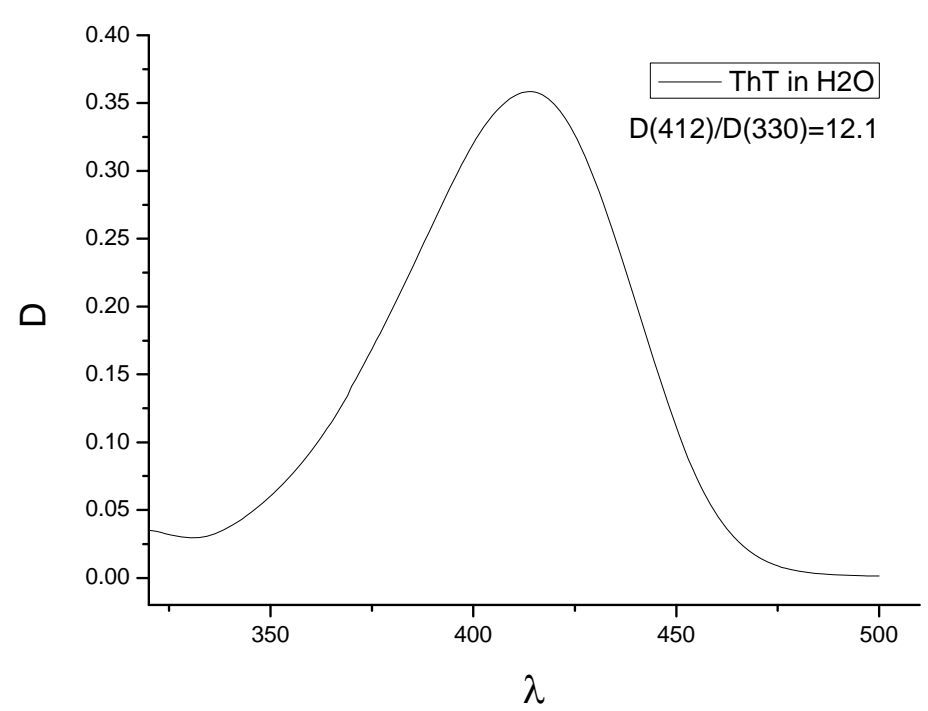

Figure SI14. Absorbance spectrum of ThT in aqueous solution. Ratio $\mathrm{D}_{412} / \mathrm{D}_{330}$ for ThT sample is $\sim 12.1$ indicating low amount of ThT degradation products. 


\section{Invariance of electron transition properties for charged molecule relative to a coordinate origin displacement}

Consider molecule as a system of charges $q_{\alpha}$ (in atomic system of units $q_{\alpha}=Z_{\mathrm{A}}$ for nuclei and $q_{\alpha}=-1$ for electrons) with coordinates $\boldsymbol{r}_{\alpha}$, located in external electric field with potential $\varphi(\mathbf{r})$. Let potential $\varphi(\mathbf{r})$ changes slightly within space occupied by the molecule, then potential energy of the molecule $\sum_{\alpha} q_{\alpha} \varphi\left(\boldsymbol{R}+\boldsymbol{r}_{\alpha}\right)$ can be expanded in powers of $\boldsymbol{r}_{\alpha}$ for any arbitrarily chosen point $\boldsymbol{R}$ within the molecule [2]:

$$
\sum_{\alpha} q_{\alpha} \varphi\left(\boldsymbol{R}+\boldsymbol{r}_{\alpha}\right)=\sum_{\alpha} q_{\alpha} \varphi(\boldsymbol{R})+\sum_{\alpha} q_{\alpha} \nabla \varphi \boldsymbol{r}_{\alpha}=\mathrm{Q} \varphi(\boldsymbol{R})-\boldsymbol{D E},
$$

where total charge of the molecule $\mathrm{Q}=\sum_{\alpha} q_{\alpha}=\sum_{A} Z_{A}-\sum_{i} 1=\sum_{A} Z_{A}-\mathrm{N}$ (N - number of electrons), dipole moment $\boldsymbol{D}=\sum_{\alpha} q_{\alpha} \boldsymbol{r}_{\alpha}=\sum_{A} Z_{A} \boldsymbol{R}_{A}-\sum_{i} \boldsymbol{r}_{\mathrm{i}},\left(\boldsymbol{R}_{A}-\right.$ nuclei coordinates, $\boldsymbol{r}_{i}-$ electron coordinates), $\boldsymbol{E}=-\nabla \varphi$ is external electric field, homogenious within molecular extent.

According to Eq.(1) energy correction has two terms and, since the first term $Q \varphi(R)$ is constant, the effect of environment on the molecule energy will be related to change in the second item $-\boldsymbol{D E}$. The latter using quantum mechanics notation gives correcting hamiltonian

$$
\hat{\mathrm{H}}^{\prime}=-\hat{\mathbf{D}} \boldsymbol{E} \text {. }
$$

It is important to note that at quantum-chemical calculations of charged molecular systems $(\mathrm{Q} \neq 0)$ one encounters the difficulty (see [3]), related to non-invariance in magnitudes of multipole moments of orders $\mathrm{n} \geq 1$ relative to displacement $\boldsymbol{a}$ of coordinates origin. Dipole moment value as well as energy correction term $\mathrm{E}^{\prime}$ defined on its base will change in a following way

$$
\begin{aligned}
& <\Psi|\hat{\mathbf{D}}| \Psi>\rightarrow<\Psi|\hat{\mathbf{D}}| \Psi>+\mathrm{Q} \mathbf{a}, \\
& <\Psi\left|\hat{\mathrm{H}}^{\prime}\right| \Psi>\rightarrow<\Psi\left|\hat{\mathrm{H}}^{\prime}\right| \Psi>-\mathrm{Q}(\mathbf{a E}) .
\end{aligned}
$$

Choice of coordinates origin and related to it problems were discussed earlier [4].

Here we would like to point out that changes of dipole moment and transition energy between electronic levels do not depend on choice of coordinates system. Indeed, for $\Psi_{0} \rightarrow \Psi$ transition (e.g. from the ground singlet state $S_{0}$ to the excited singlet state $S_{1}$ ) change of dipole moment $\Delta \mathbf{D}$ will be:

$$
\Delta \mathbf{D}=<\Psi|\hat{\mathbf{D}}| \Psi>-<\Psi_{0}|\hat{\mathbf{D}}| \Psi_{0}>\rightarrow<\Psi|\hat{\mathbf{D}}| \Psi>-<\Psi_{0}|\hat{\mathbf{D}}| \Psi_{0}>+ \text { Qa }-Q \mathbf{a}=\text { inv } .
$$

The same behavior fulfils for energy transition $\Delta \mathrm{E}$ :

$$
\begin{aligned}
& \Delta \mathrm{E}=<\Psi\left|\hat{\mathrm{H}}+\hat{\mathrm{H}}^{\prime}\right| \Psi>-<\Psi_{0}\left|\hat{\mathrm{H}}+\hat{\mathrm{H}}^{\prime}\right| \Psi_{0}>=<\Psi|\hat{\mathrm{H}}| \Psi>-<\Psi_{0}|\hat{\mathrm{H}}| \Psi_{0}>-(\Delta \mathbf{D E}) \rightarrow \\
& <\Psi|\hat{\mathrm{H}}| \Psi>-<\Psi_{0}|\hat{\mathrm{H}}| \Psi_{0}>-(\Delta \mathbf{D E})-\mathrm{Q}(\mathbf{a E})+\mathrm{Q}(\mathbf{a E})=\text { inv. }
\end{aligned}
$$

We can note as well that oscillator strength for $\Psi_{0} \rightarrow \Psi$ transition, calculated using matrix element $<\Psi_{0}|\hat{\mathbf{D}}| \Psi>$, is invariant relative to coordinates origin displacement a due to orthogonality of functions $\Psi_{0}$ and $\Psi,\left\langle\Psi_{0}\right| \Psi>=0$ :

$$
<\Psi_{0}|\hat{\mathbf{D}}| \Psi>\rightarrow<\Psi_{0}|\hat{\mathbf{D}}| \Psi>+\mathbf{a}<\Psi_{0} \mid \Psi>=\text { inv. }
$$




\section{References}

1. Williams, A.T.R., S.A. Winfield, and J.N. Miller, Relative fluorescence quantum yields using a computer-controlled luminescence spectrometer. Analyst, 1983. 108(1290): p. 1067-1071.

2. Landau, L. and E. Lifshits, Field theory. 1973, Moscow: Nauka.

3. Kaplan, I., Introduction to intermolecular interaction theory. 1987, Moscow: Nauka. 341.

4. http://www.ccl.net/chemistry/resources/messages/1995/01/13.002-dir/index.html. 